\title{
Next-generation vaccine platforms for COVID-19
}

\author{
Consensus among experts is that only an effective COVID-19 vaccine will end the pandemic. This Comment \\ focuses on how this pandemic has accelerated the development of vaccine platforms distinct from classical \\ vaccines; these novel platforms may also increase the response time when new viruses emerge in the future.
}

\section{Debby van Riel and Emmie de Wit}

A n outbreak of highly pathogenic avian influenza (HPAI) virus of the $\mathrm{H} 5 \mathrm{~N} 1$ subtype was diagnosed in Hong Kong in 1997, with 18 human cases including six deaths. This was the first known outbreak of influenza A virus resulting from direct transmission of an avian influenza virus from chickens to humans without an intermediate reservoir such as pigs ${ }^{1}$. This outbreak increased the awareness of the risk of a devastating pandemic, and showed that more resources should be diverted to pandemic preparedness planning ${ }^{2}$. Many considered this an overblown response to a small outbreak, aimed at acquiring more research funding, especially when years went by without an $\mathrm{H} 5 \mathrm{~N} 1$ pandemic, despite the widespread, continued circulation of HPAI $\mathrm{H} 5 \mathrm{~N} 1$ in poultry with regular spillover to humans, resulting in more than 850 human cases and over 450 deaths to date ${ }^{3}$. The emergence of severe acute respiratory syndrome coronavirus (SARS-CoV) in 2002 put everyone on alert for a while, but this pandemic was contained through old-fashioned contact tracing and isolation procedures after causing $>8,000$ cases in 27 countries $^{4}$. Subsequently, the $2009 \mathrm{H} 1 \mathrm{~N} 1$ pandemic turned out to be relatively mild, with a low case fatality rate ${ }^{5}$. The Ebola virus epidemic in West Africa in 2013-2016 was a turning point. Whereas the largest previous Ebola virus outbreaks had resulted in several hundred cases in confined areas, this epidemic resulted in close to 30,000 cases in ten countries and took more than three years to bring under control ${ }^{6}$. The unprecedented number of cases, geographic spread, and enormous amount of money and effort needed to end this epidemic made clear that zoonotic viruses pose an immense threat to global health and economies. Efforts were launched to identify viruses with epidemic potential ${ }^{7}$, and money was invested in developing vaccines against some of these, as well as in the development of new, rapid vaccine platforms. Now, six months after the discovery of SARS-CoV-2, antivirals and vaccines are in development, with many treatment options and vaccines in clinical trials worldwide. Even though antivirals are important to dampen the disease burden of the current pandemic, effective vaccines are essential to control it. The World Health Organization (WHO) estimates that there are 133 COVID-19 vaccines in development ${ }^{8}$. Many of these are novel platforms with little pre-existing data on safety and efficacy in humans.

\section{Classic vaccine platforms}

The vast majority of vaccines currently licensed for human use can be divided into virus-based or protein-based vaccines (Fig. 1). The virus-based vaccines can consist of inactivated virus that is no longer infectious, or live-attenuated virus. Since whole-inactivated viruses do not replicate, adjuvants are required to stimulate the immune system. Live-attenuated virus vaccines are classically generated by passaging in cell culture until it loses its pathogenic properties and causes only a mild infection upon injection. Protein-based vaccines can consist of a protein purified from the virus or virus-infected cells, recombinant protein or virus-like particles. Virus-like particles consist of the structural viral proteins necessary to form a virus particle, but lack the viral genome and non-structural proteins. Protein-based vaccines require the addition of an adjuvant to induce a strong immune response. Two COVID-19 vaccines based on these classical platforms are currently in clinical trials, one based on whole-inactivated virus and one consisting of recombinant protein (Fig. 1).

These classical vaccine platforms have contributed to major public health breakthroughs, such as the eradication of smallpox and a vaccine to prevent cancer ${ }^{9,10}$. However, certain limitations are associated with several of these platforms that make them less amenable to fast vaccine production in a pandemic. In the case of SARS-CoV-2, large quantities of virus would need to be grown under biosafety level 3 (BSL3) conditions for a whole-inactivated vaccine; extensive safety testing is required to ensure live-attenuated viruses are safe and do not easily revert to wild type, and several recombinant proteins need to be produced simultaneously for virus-like particle vaccines.

\section{Next-generation vaccine platforms}

The main advantage of next-generation vaccines is that they can be developed based on sequence information alone. If the viral protein(s) important to provide protection from infection or disease, and thus for inclusion in a vaccine (that is, the vaccine antigen), is known the availability of coding sequences for this viral protein(s) suffices to start vaccine development, rather than having to depend on the ability to culture the virus. This makes these platforms highly adaptable and speeds up vaccine development considerably, as is clear from the fact that the majority of COVID-19 vaccine clinical trials currently ongoing involve a next-generation platform (Fig. 1).

For COVID-19, several viral vector, nucleic acid-based vaccines and antigen-presenting cells are in (pre) clinical development (Fig. 1). Viral vector vaccines consist of a recombinant virus (that is, the viral vector), often attenuated to reduce its pathogenicity, in which genes encoding viral antigen(s) have been cloned using recombinant DNA techniques. Vector vaccines can either be replicating or non-replicating. Replicating vector vaccines infect cells in which the vaccine antigen is produced as well as more infectious viral vectors able to infect new cells that will then also produce the vaccine antigen. Non-replicating vector vaccines initially enter cells and produce the vaccine antigen, but no new virus particles are formed. Because viral vector vaccines result in endogenous antigen production, both humoral and cellular immune responses are stimulated. One advantage of these viral vector-based vaccines is therefore that a single dose can be sufficient for protection, as in the case of the vesicular-stomatitis virus-based Ervebo vaccine against Ebola virus ${ }^{11}$.

Nucleic acid-based vaccines can consist of DNA or mRNA and can be adapted quickly when new viruses emerge, which 
Classical platforms

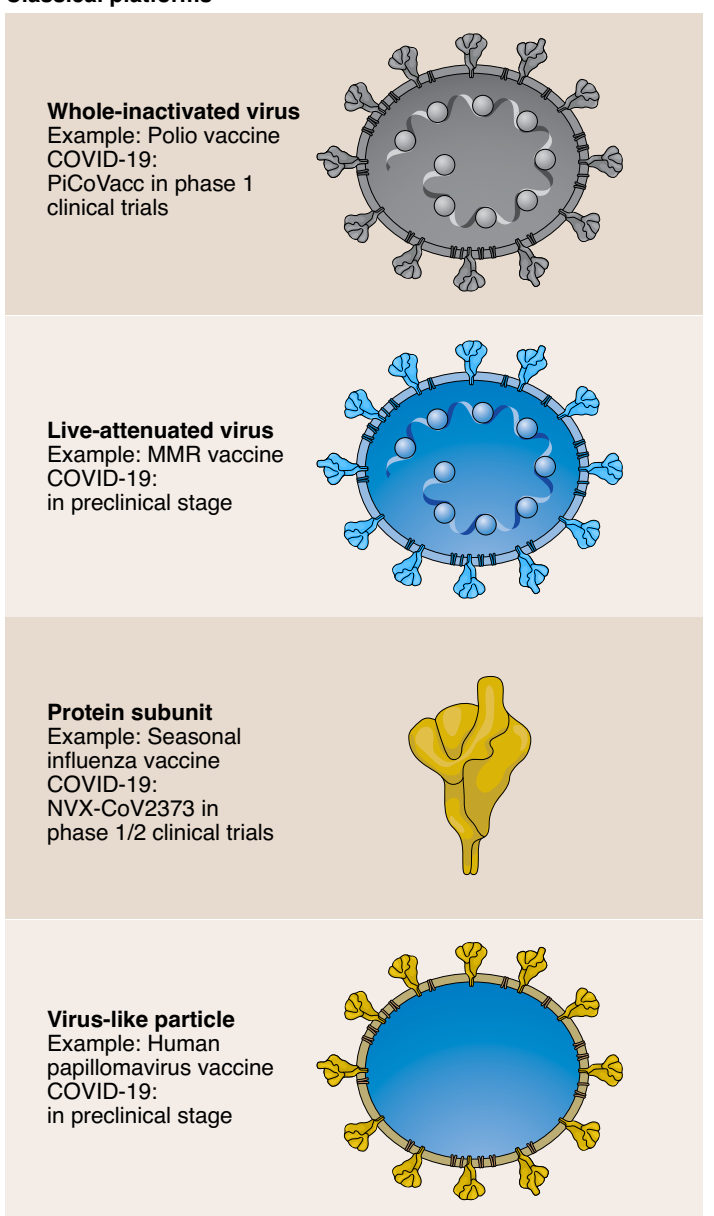

Next-generation platforms

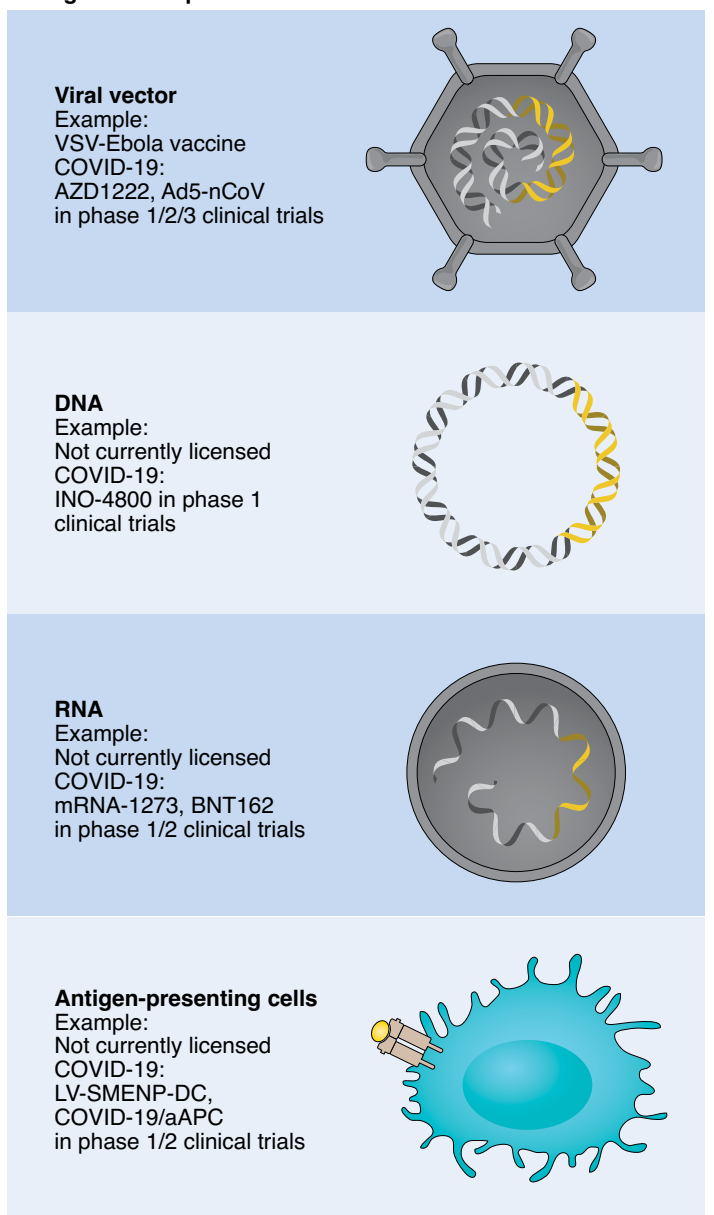

Fig. 1| An overview of the different vaccine platforms in development against COVID-19. A schematic representation is shown of the classical vaccine platforms that are commonly used for human vaccines, and next-generation platforms, where very few have been licensed for use in humans. The stage of development for each of these vaccine platforms for COVID-19 vaccine development is shown; online vaccine trackers are available to follow these vaccines through the clinical development and licensing process ${ }^{21}$.

is why these were among the very first COVID-19 vaccines to enter clinical trials. DNA vaccines consist of a synthetic DNA construct encoding the vaccine antigen. For efficient uptake of the construct into cells, injection needs to be followed by electroporation. After uptake into cells, the vaccine antigen is expressed from the DNA construct. mRNA-based vaccines work on the same principle as DNA vaccines, except that the first steps (nuclear translocation of the DNA construct and transcription into mRNA) are bypassed. Self-replicating RNA vaccines are likely to induce protective immunity using a lower dose, because more vaccine antigen is expressed per cell ${ }^{12}$. Since mRNA is not very stable, these constructs include modified nucleosides to prevent degradation. A carrier molecule is necessary to enable entry of the mRNA into cells; lipid nanoparticles are most commonly used. Nucleic acid-based vaccines induce a humoral and cellular immune response, but multiple doses are required.

Antigen-presenting cells are an essential component in the immune system's response to a vaccine. Loading antigen-presenting cells with peptides that would otherwise be produced by vaccination bypasses the first steps after vaccination. Traditionally, dendritic cells are harvested from the individual, then expanded and manipulated to present the desired antigen, and infused back into the same individual. This is cost-prohibitive and too time-consuming for a vaccine deployed on a large scale. This has led to the development of artificial antigen-presenting cells, where immortalized cells are transduced with lentiviruses to effectively mimic antigen-presenting cells, as is the case for COVID-19/aAPC. Extra cold-chain requirements for a cell-based vaccine and infusion procedures hamper the deployment of these vaccines on a large scale, even more so since multiple doses are required for an efficient response. COVID-19 vaccines based on all next-generation platforms are currently in clinical trials; some of these have already moved from phase 1 into phase 2 or $2 / 3$ (Fig. 1).

\section{Vaccine requirements and challenges}

While the development of vaccines against COVID-19 is ongoing, it is important to define what we expect from this vaccine, or vaccines for future emerging viruses. Needless to say, the vaccine should be safe and effective, and should not induce enhanced disease upon subsequent infection, whether through vaccine-associated enhanced respiratory disease or antibody-dependent enhancement, as has been observed with certain SARS-CoV vaccines in animal models in the past ${ }^{13}$. In order to prevent 
severe disease after infection, vaccination should result in either (1) complete abrogation or significant reduction of transmission within the population by the induction of herd immunity or (2) prevention of severe disease in all vaccinated individuals. Both approaches would require the production of large quantities of vaccine, distributed worldwide. A single dose vaccine that would not require a cold chain would contribute to the timeframe in which large-scale, global vaccination can be achieved. Ideally, vaccination would induce long-lived immunity, but annual vaccination would be feasible based on experiences with the annual influenza vaccine. Vaccination campaigns that either induce herd immunity or protect vaccinated individuals from severe disease face different challenges. Herd immunity for SARS-CoV-2 would require vaccination of $\sim 67 \%$ of the population ${ }^{14}$, which is an average and would not prevent clusters of susceptible individuals. However, in recent years vaccine hesitance, identified as a major threat to global health by the $\mathrm{WHO}^{15}$, increased in many countries, and a recent study showed that $26 \%$ of the French population would not take a SARS-CoV-2 vaccine $^{16}$. Vaccination that does not abrogate transmission but does result in protection from severe disease seems more straightforward. However, two important risk groups for developing severe COVID19 , elderly ( $>65$ years old) and obesity (body mass index $>40$ ) have previously been linked to reduced vaccine efficacy using classical vaccination approaches ${ }^{17,18}$. Whether new vaccine platforms have an increased immunogenicity in these risk groups compared to classical vaccination approaches remains to be determined.

Besides the question of efficacy for any of the COVID-19 vaccines under development, a major hurdle will be large-scale manufacturing. Since the next-generation platforms, with the exception of two viral vector-based vaccines (Dengvaxia and Ervebo) are not licensed for use in humans, the feasibility to rapidly manufacture these on a large-scale is currently unclear ${ }^{19}$. Although vaccine manufacturing capacity exists for the classical platforms, using the existing infrastructure would potentially go at the expense of regular vaccine production. Maintaining vaccination status for all vaccine-preventable diseases, while at the same time producing hundreds of millions of doses of COVID-19 vaccines, will be essential for global health. Besides the vaccine antigen itself, the required adjuvants or delivery molecules and, in the case of DNA vaccines, special delivery devices, will need to be manufactured on a mass scale as well. To be of use, hundreds of millions of doses need to be manufactured and distributed. Moreover, with the potential exception of DNA vaccines, all vaccines require a cold chain for distribution. Therefore, many international initiatives and investments are currently made to increase the capacity to produce and distribute vaccines. These collaborative programmes will be crucial for the large-scale deployment of vaccines to contain the COVID-19 pandemic; global distribution to prevent disparities in vaccination programmes between high-income countries and the rest of the world is essential in this effort.

\section{Future directions}

The development of many of the next-generation platforms described here has so far been driven mainly by their potential use in cancer therapies. The COVID-19 pandemic has fast-tracked their development as vaccine platforms for emerging viruses. If current predictions become reality, the first vaccines against COVID-19 will be licensed within a year. These licensed vaccines are likely to include some of the next-generation platforms described here. This in itself will be a major public health achievement, yet will simultaneously result in a permanent change to the vaccine platform landscape and an increased vaccine manufacturing capacity for these novel platforms. Plans should be developed to ensure that the large-scale manufacturing infrastructure being built now to respond to the COVID-19 pandemic is maintained for potential future vaccine needs, as has been done for influenza vaccines ${ }^{20}$.

Once next-generation platforms are licensed, their use for other pathogens or disease indications are likely to become more easily attainable. Since these platforms only require sequence information to initiate vaccine development, this will increase the flexibility to adapt vaccines to antigenic changes in circulating strains, and to newly emerging viruses in general. The wider array of possibilities for pre-emptive and reactive vaccine design, as well as faster development and manufacturing options, will permanently change our ability to rapidly respond to emerging viruses. As such, the investments made now in vaccine platform development and manufacturing will pay off when we are able to respond even faster when a new virus emerges in the future.

\section{Debby van Riel ${ }^{1}$ and Emmie de Wit (D) $2 \bowtie$} ${ }^{1}$ Department of Viroscience, Erasmus MC, Rotterdam, the Netherlands. ${ }^{2}$ Laboratory of Virology, National Institute of Allergy and Infectious Diseases, National Institutes of Health, Hamilton, MT, USA.

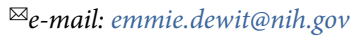

Published online: 23 July 2020

https://doi.org/10.1038/s41563-020-0746-0

References

1. Chan, P. K. S. Clin. Infect. Dis. 34, S58-S64 (2002).

2. Iskander, J., Strikas, R. A., Gensheimer, K. F., Cox, N. J. \& Redd, S. C. Emerg. Infect. Dis. 19, 879-885 (2013).

3. Cumulative Number of Confirmed Human Cases for Avian Influenza A(H5N1) Reported to WHO, 2003-2020 (WHO, 2020); https://www.who.int/influenza/human_animal_interface/2020_ MAY_tableH5N1.pdf?ua=1

4. Summary of Probable SARS Cases with Onset of Illness from 1 November 2002 to 31 July 2003 (WHO, 2003); https://www.who. int/csr/sars/country/table2004_04_21/en/

5. Wong, J. Y. et al. Epidemiology 24, 830-841 (2013).

6. Bausch, D. G. Curr. Top. Microbiol. Immunol. 411, 63-92 (2017).

7. Mehand, M. S., Al-Shorbaji, F., Millett, P. \& Murgue, B. Antiviral Res. 159, 63-67 (2018).

8. DRAFT Landscape of COVID-19 Candidate Vaccines (WHO, 2020); https://www.who.int/who-documents-detail/ draft-landscape-of-covid-19-candidate-vaccines

9. Greenwood, B. Philos. Trans. R. Soc. Lond. B 369, 20130433 (2014).

10. Patel, C. et al. Euro. Surveill. 23, 30-40 (2018).

11. Henao-Restrepo, A. M. et al. Lancet 389, 505-518 (2017).

12. Vogel, A. B. et al. Mol. Ther. 26, 446-455 (2018).

13. Graham, B. S. Science 368, 945-946 (2020).

14. Randolph, H. E. \& Barreiro, L. B. Immunity 52, 737-741 (2020).

15. Ten Threats to Global Health in 2019 (WHO, 2019); https://www.who.int/news-room/feature-stories/ ten-threats-to-global-health-in-2019

16. Coconel Group Lancet Infect. Dis. 20, 769-770 (2020). 17. Ciabattini, A. et al. Semin. Immunol. 40, 83-94 (2018). 18. Sheridan, P. A. et al. Int. J. Obes. 36, 1072-1077 (2012).

19. Thanh Le, T. et al. Nat. Rev. Drug Discov. 19, 305-306 (2020).

20. McLean, K. A., Goldin, S., Nannei, C., Sparrow, E. \& Torelli, G. Vaccine 34, 5410-5413 (2016).

21. Craven, J. COVID-19 Vaccine Tracker (RAPS, 2020);

https://www.raps.org/news-and-articles/news-articles/2020/3/ covid-19-vaccine-tracker

\section{Acknowledgements}

The authors would like to thank V. Munster (National Institute of Allergy and Infectious Diseases, National Institutes of Health) for critically reading the manuscript and R. Kissinger (National Institute of Allergy and Infectious Diseases, National Institutes of Health) for preparing the figure. D.v.R. is supported by the Netherlands Organization for Scientific Research (grant number 91718308) and a EUR fellowship. E.d.W. is supported by the Intramural Research Program of National Institute of Allergy and Infectious Diseases, National Institutes of Health. 